\title{
Characteristics of Dark Matter
}

\author{
Manu Mitra* \\ Department of Electrical Engineering, Alumnus of University of Bridgeport, United States
}

*Corresponding author: Manu Mitra, Department of Electrical Engineering, Alumnus of University of Bridgeport, United States

Received: 鴜 May 03, 2019

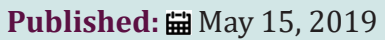

\begin{abstract}
There are many theories about the Dark Matter. It is one of complex part in Astrophysics to understand its behavior, nature and its complex performance. In this research paper, various characteristics are taken to plot the graphs based on experiments, projection, and theoretical point of view; various parameters are taken into consideration such as spin-independent and spindependent. $\mathrm{X}$ axis is plotted with lower bound in $\mathrm{GeV} / \mathrm{c}^{2}$. Y axis is plotted in variable cross section of $10^{-54} \mathrm{~cm}^{2}$ and $10^{-26} \mathrm{~cm}^{2}$ and other parameters illustrated in the table.
\end{abstract}

Keywords: Dark Matter; Characteristics; Spin-independent; Spin-dependent

\section{Introduction}

To make sense of dataset and theoretical representation for the universe; first, standard model of cosmology i.e., Cold Dark Matter that requires around $96 \%$ of the universe is composed of unknown material that cannot be directly observed. Second requirement for dark matter is more grounded in sound observation and classical physics. Around two thirds of this unknown material can't possibly be matter, one of the reasons is that it grows as universe grows so we call it as "dark energy". The remaining section we call dark matter since it is capable of generating gravity. In this specific context, dark matter is raised to balance the math - within a set of formulae which are straining around $96 \%$ of the universe is invisible and undetectable. In the second theory; the way galaxies clusters and interact dynamically, it signifies that they are composed of only visible and other known matter that lie within them. Milky
Way itself is rotating in a manner that would result flying apart, if there wasn't additional invisible matter generating additional gravitational phenomenon. That is the reasons to think that there could be something else that is unknown [1].

\section{Literature Review}

If Academics and scientists cannot see dark matter, then how do they know they exists? Scientist calculate mass of large objects in space by comprehending their motion. Expert Astronomers examine spiral galaxies to view materials in the center moving faster than on the outer edges. Instead they found that stars in both locations travelled at the same velocity, demonstrating that galaxies contained more mass than could be seen. Clusters of galaxies would retrogress [2]. if the only mass they contained were visible to conventional astronomical measurements. (Figures 1-3)

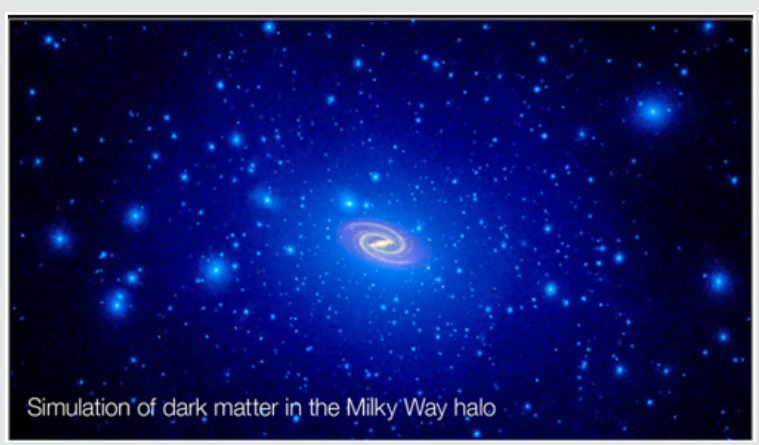

Figure 1: Illustrates simulation of dark matter in the Milky Way halo. Image Reference: Nola Taylor Redd [2]. 


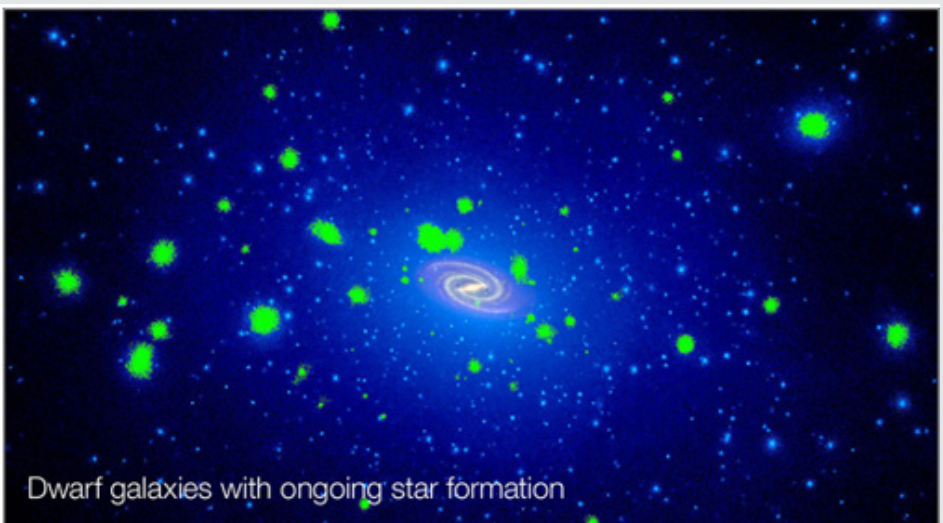

Figure 2: Illustrates Dwarf galaxies with ongoing star formation. Image Reference: Nola Taylor Redd [2].

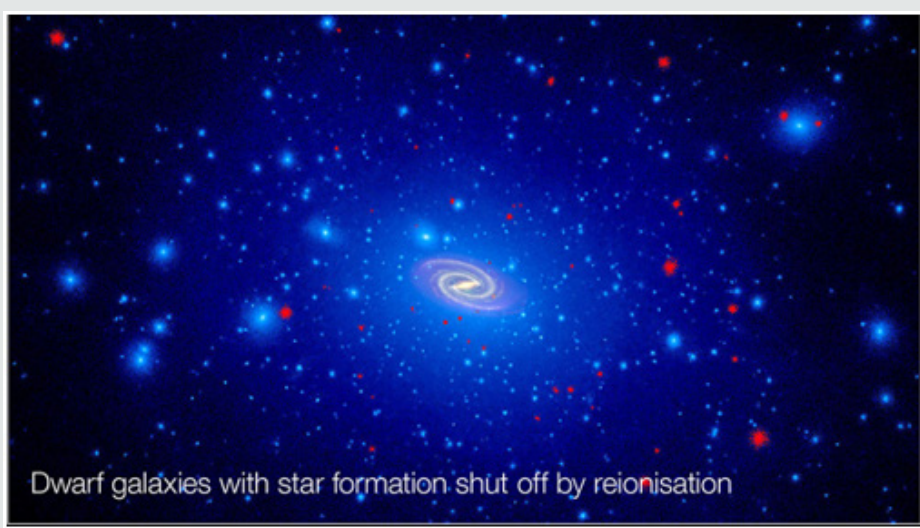

Figure 3: Illustrates simulation of Dwarf galaxies with star formation shut off by reionization. Image Reference: Nola Taylor Redd [2].

\section{Dark Matter Simulations}

a) Dark matter for spin dependent

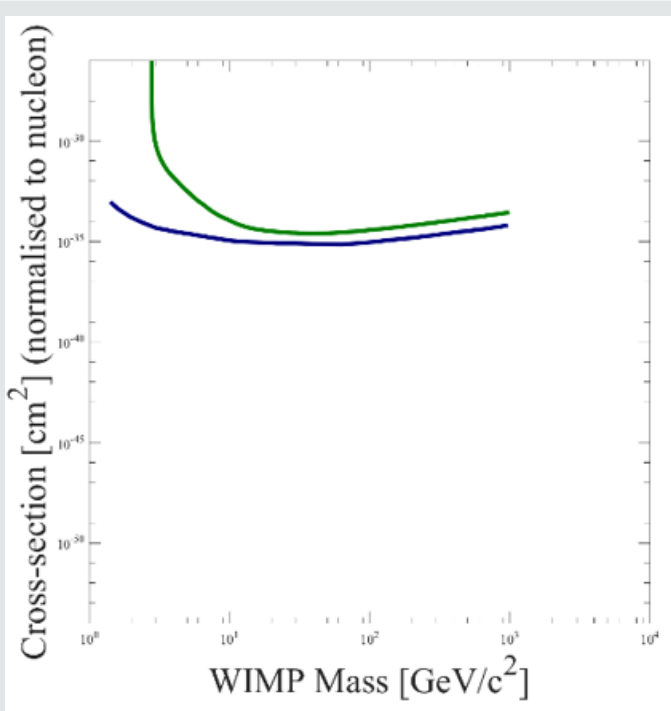

Figure 4: Illustrates characteristics of Dark Matter for Spin dependent for Experiments.
A simulation for Dark Matter was performed for Spin dependent [3], (Figures 4-6).

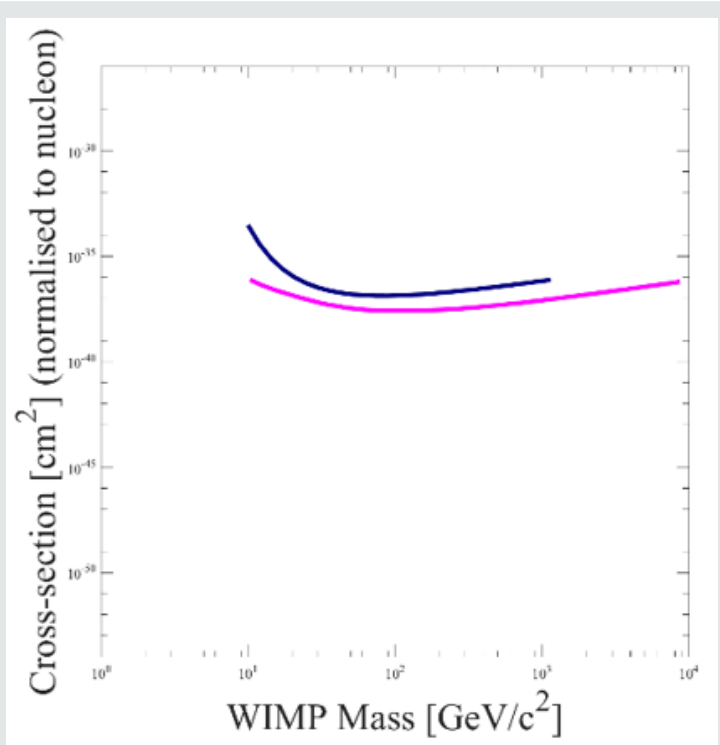

Figure 5: Illustrates characteristics of Dark Matter for Spin dependent for Projection. 


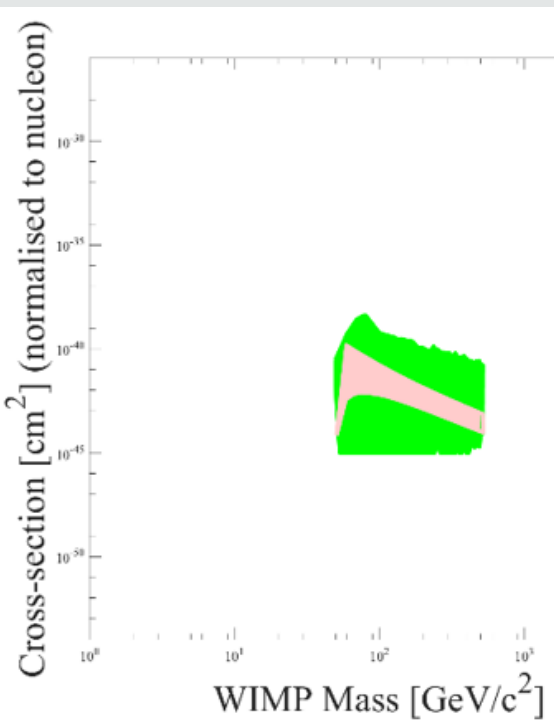

Figure 6: Illustrates characteristics of Dark Matter for Spin dependent for Theory.

b) Dark matter for spin independent

A simulation for Dark Matter was performed for Spin independent (Figures 7-9), (Table 1-20).

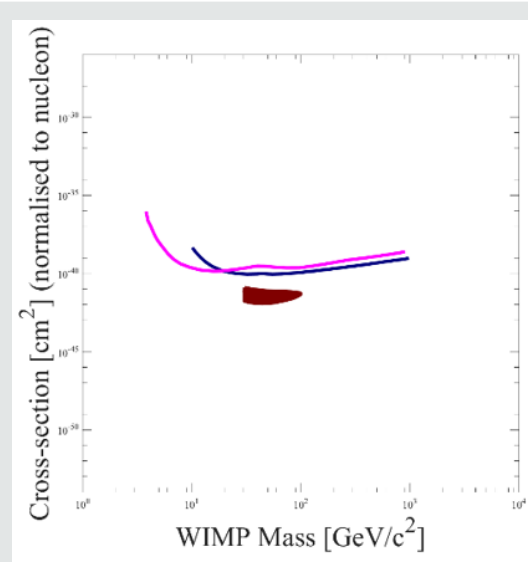

Figure 7: Illustrates characteristics of Dark Matter for Spin independent for Experiments.

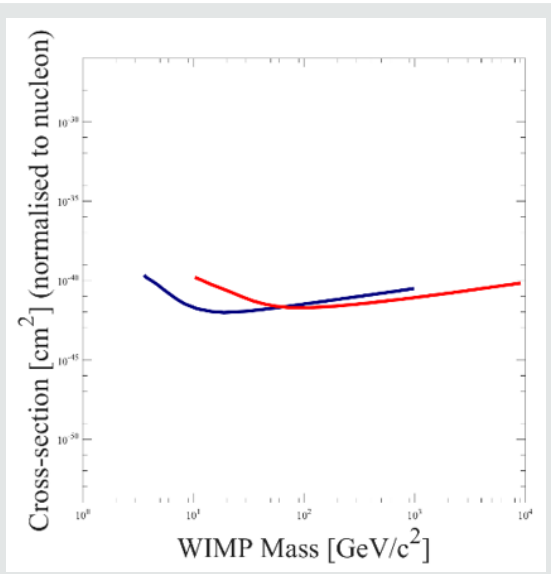

Figure 8: Illustrates characteristics of Dark Matter for Spin independent for Projection.

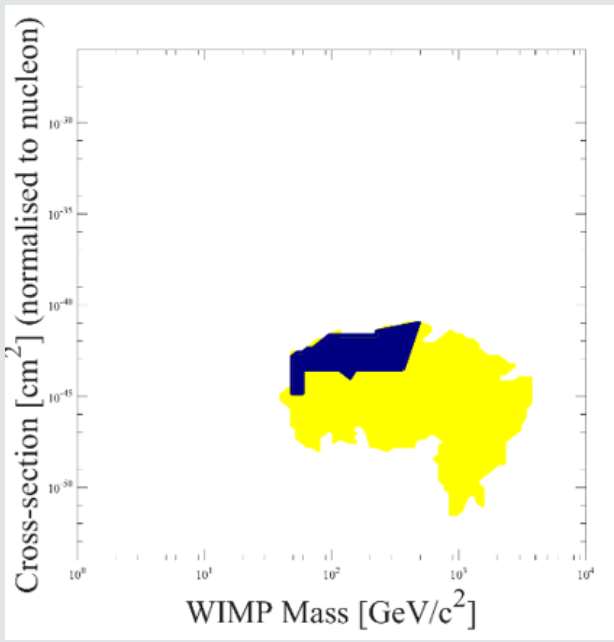

Figure 9: Illustrates characteristics of Dark Matter for Spin independent for Theory.

Table 1: Simulation Notes for Dark Matter Characteristics for Spin dependent.

\begin{tabular}{|c|c|c|}
\hline SI No: & Simulation Specifications & Value \\
\hline 1 & x range (WIMP mass) & 1 (lower bound) \\
\hline 2 & x range (WIMP mass) & $\begin{array}{c}10000 \text { (upper bound) GeV/ } \\
\mathrm{c}^{\wedge} 2\end{array}$ \\
\hline 3 & $\begin{array}{c}\text { y range (cross section) } \\
\text { smallest }\end{array}$ & c-s: $10^{\wedge}-54 \mathrm{~cm}^{\wedge} 2$ \\
\hline 4 & $\begin{array}{c}\text { y range (cross section) } \\
\text { largest }\end{array}$ & c-s: $10^{\wedge}-26 \mathrm{~cm}^{\wedge} 2$ \\
\hline 5 & $\begin{array}{c}\text { Type of method } \\
\text { Experiment }\end{array}$ \\
\hline 6 & Limit & Spin-dependent \\
\hline 7 & Year & Greatest Hits \\
\hline 8 & Simulation Type & 2001 \\
\hline
\end{tabular}

Table 2: Simulation Notes for Dark Matter Characteristics for Spin dependent.

\begin{tabular}{|c|c|c|}
\hline Sl No: & Simulation Specifications & Value \\
\hline 1 & $\mathrm{x}$ range (WIMP mass) & 1 (lower bound) \\
\hline 2 & $\mathrm{x}$ range (WIMP mass) & 10000 (upper bound) $\mathrm{GeV} /$ \\
\hline 3 & $\begin{array}{c}\text { y range (cross section) } \\
\text { smallest }\end{array}$ & c-s: $10^{\wedge}-54 \mathrm{~cm}^{\wedge} 2$ \\
\hline 4 & $\begin{array}{c}\text { y range (cross section) } \\
\text { largest }\end{array}$ & c-s: $10^{\wedge}-26 \mathrm{~cm}^{\wedge} 2$ \\
\hline 5 & Type of method & Projection \\
\hline 6 & Limit & Spin-dependent \\
\hline 7 & Year & All \\
\hline 8 & Simulation Type & Greatest Hits \\
\hline
\end{tabular}


Table 3: Simulation Notes for Dark Matter Characteristics for Spin dependent.

\begin{tabular}{|c|c|c|}
\hline Sl No: & Simulation Specifications & Value \\
\hline 1 & $\mathrm{x}$ range (WIMP mass) & 1 (lower bound) \\
\hline 2 & $\mathrm{x}$ range (WIMP mass) & $\begin{array}{c}10000 \text { (upper bound) } \mathrm{GeV} / \\
\mathrm{c}^{\wedge} 2\end{array}$ \\
\hline 3 & $\begin{array}{c}\text { y range (cross section) } \\
\text { smallest }\end{array}$ & c-s: $10^{\wedge}-54 \mathrm{~cm}^{\wedge} 2$ \\
\hline 4 & $\begin{array}{c}\text { y range (cross section) } \\
\text { largest }\end{array}$ & c-s: $10^{\wedge}-26 \mathrm{~cm}^{\wedge} 2$ \\
\hline 5 & Type of method & Theory \\
\hline 6 & Limit & Spin-dependent \\
\hline 7 & Year & 2000 \\
\hline 8 & Simulation Type & Greatest Hits \\
\hline
\end{tabular}

Table 4: Simulation Notes for Dark Matter Characteristics for Spin Independent.

\begin{tabular}{|c|c|c|}
\hline SI No: & Simulation Specifications & Value \\
\hline 1 & $\mathrm{x}$ range (WIMP mass) & 1 (lower bound) \\
\hline 2 & $\mathrm{x}$ range (WIMP mass) & 10000 (upper bound) $\mathrm{GeV} /$ \\
\hline 3 & $\begin{array}{c}\text { y range (cross section) } \\
\text { smallest }\end{array}$ & c-s: $10^{\wedge}-54 \mathrm{~cm}^{\wedge} 2$ \\
\hline 4 & $\begin{array}{c}\text { y range (cross section) } \\
\text { largest }\end{array}$ & c-s: $10^{\wedge}-26 \mathrm{~cm}^{\wedge} 2$ \\
\hline 5 & Type of method & Experiment \\
\hline 6 & Limit & Spin-independent \\
\hline 7 & Year & 2000 \\
\hline 8 & Simulation Type & Greatest Hits \\
\hline
\end{tabular}

Table 5: Simulation Notes for Dark Matter Characteristics for Spin Independent.

\begin{tabular}{|c|c|c|}
\hline SI No: & Simulation Specifications & Value \\
\hline 1 & $\mathrm{x}$ range (WIMP mass) & 1 (lower bound) \\
\hline 2 & $\mathrm{x}$ range (WIMP mass) & $\begin{array}{c}10000 \text { (upper bound) } \mathrm{GeV} / \\
\mathrm{c}^{\wedge} 2\end{array}$ \\
\hline 3 & $\begin{array}{c}\text { y range (cross section) } \\
\text { smallest }\end{array}$ & c-s: $10^{\wedge}-54 \mathrm{~cm}^{\wedge} 2$ \\
\hline 4 & $\begin{array}{c}\text { y range (cross section) } \\
\text { largest }\end{array}$ & c-s: $10^{\wedge}-26 \mathrm{~cm}^{\wedge} 2$ \\
\hline 5 & Type of method & Projection \\
\hline 6 & Limit & Spin-independent \\
\hline 7 & Year & 2000 \\
\hline 8 & Simulation Type & Greatest Hits \\
\hline
\end{tabular}

Table 6: Simulation Notes for Dark Matter Characteristics for Spin Independent.

\begin{tabular}{|c|c|c|}
\hline SI No: & Simulation Specifications & Value \\
\hline 1 & x range (WIMP mass) & 1 (lower bound) \\
\hline 2 & x range (WIMP mass) & $\begin{array}{c}10000 \text { (upper bound) GeV/ } \\
\mathrm{c}^{\wedge} 2\end{array}$ \\
\hline 3 & $\begin{array}{c}\text { y range (cross section) } \\
\text { smallest }\end{array}$ & c-s: $10^{\wedge}-54 \mathrm{~cm}^{\wedge} 2$ \\
\hline 4 & $\begin{array}{c}\text { y range (cross section) } \\
\text { largest }\end{array}$ & c-s: $10^{\wedge}-26 \mathrm{~cm}^{\wedge} 2$ \\
\hline 5 & $\begin{array}{c}\text { Type of method } \\
\text { Theory }\end{array}$ \\
\hline 6 & Limit & Spin-independent \\
\hline 7 & Year & All \\
\hline 8 & Simulation Type & \\
\hline
\end{tabular}

Table 7: Simulation Notes for Dark Matter Characteristics for CDEX-10.

\begin{tabular}{|c|c|c|}
\hline SI No: & Simulation Specifications & Value \\
\hline 1 & x range (WIMP mass) & 1 (lower bound) \\
\hline 2 & x range (WIMP mass) & $\begin{array}{c}10000 \text { (upper bound) } \mathrm{GeV} / \\
\mathrm{c}^{\wedge} 2\end{array}$ \\
\hline 3 & $\begin{array}{c}\text { y range (cross section) } \\
\text { smallest }\end{array}$ & c-s: $10^{\wedge}-54 \mathrm{~cm}^{\wedge} 2$ \\
\hline 4 & $\begin{array}{c}\text { y range (cross section) } \\
\text { largest }\end{array}$ & c-s: $10^{\wedge}-26 \mathrm{~cm}^{\wedge} 2$ \\
\hline 5 & $\begin{array}{c}\text { Type of method } \\
\text { Experiment }\end{array}$ \\
\hline 6 & Limit & CDEX-10 \\
\hline 7 & Year & All \\
\hline 8 & Simulation Type & All \\
\hline
\end{tabular}

Table 8: Simulation Notes for Dark Matter Characteristics for CDMS I (SUF).

\begin{tabular}{|c|c|c|}
\hline Sl No: & Simulation Specifications & Value \\
\hline 1 & x range (WIMP mass) & 1 (lower bound) \\
\hline 2 & x range (WIMP mass) & $\begin{array}{c}10000 \text { (upper bound) GeV/ } \\
\mathrm{c}^{\wedge} 2\end{array}$ \\
\hline 3 & $\begin{array}{c}\text { y range (cross section) } \\
\text { smallest }\end{array}$ & $\mathrm{c}-\mathrm{s}: 10^{\wedge}-54 \mathrm{~cm}^{\wedge} 2$ \\
\hline 4 & $\begin{array}{c}\text { y range (cross section) } \\
\text { largest }\end{array}$ & c-s: $10^{\wedge}-26 \mathrm{~cm}^{\wedge} 2$ \\
\hline 5 & $\begin{array}{c}\text { Type of method } \\
\text { Experiment }\end{array}$ \\
\hline 6 & Limit & CDEX-10 \\
\hline 7 & Year & All \\
\hline 8 & Simulation Type & \\
\hline
\end{tabular}


Table 9: Simulation Notes for Dark Matter Characteristics for CDMS II (Soudan).

\begin{tabular}{|c|c|c|}
\hline SI No: & Simulation Specifications & Value \\
\hline 1 & $\mathrm{x}$ range (WIMP mass) & 1 (lower bound) \\
\hline 2 & $\mathrm{x}$ range (WIMP mass) & $\begin{array}{c}10000 \text { (upper bound) } \mathrm{GeV} / \\
\mathrm{c}^{\wedge} 2\end{array}$ \\
\hline 3 & $\begin{array}{c}\text { y range (cross section) } \\
\text { smallest }\end{array}$ & c-s: $10^{\wedge}-54 \mathrm{~cm}^{\wedge} 2$ \\
\hline 4 & $\begin{array}{c}\text { y range (cross section) } \\
\text { largest }\end{array}$ & c-s: $10^{\wedge}-26 \mathrm{~cm}^{\wedge} 2$ \\
\hline 5 & Type of method & Experiment \\
\hline 6 & Limit & CDMS II (Soudan) \\
\hline 7 & Year & 2004 \\
\hline 8 & Simulation Type & All \\
\hline
\end{tabular}

Table 10: Simulation Notes for Dark Matter Characteristics for COSME.

\begin{tabular}{|c|c|c|}
\hline SI No: & Simulation Specifications & Value \\
\hline 1 & $\mathrm{x}$ range (WIMP mass) & 1 (lower bound) \\
\hline 2 & $\mathrm{x}$ range (WIMP mass) & 10000 (upper bound) $\mathrm{GeV} /$ \\
\hline 3 & $\begin{array}{c}\text { y range (cross section) } \\
\text { smallest }\end{array}$ & c-s: $10^{\wedge}-54 \mathrm{~cm}^{\wedge} 2$ \\
\hline 4 & $\begin{array}{c}\text { y range (cross section) } \\
\text { largest }\end{array}$ & c-s: $10^{\wedge}-26 \mathrm{~cm}^{\wedge} 2$ \\
\hline 5 & Type of method & Experiment \\
\hline 6 & Limit & COSME \\
\hline 7 & Year & All \\
\hline 8 & Simulation Type & All \\
\hline
\end{tabular}

Table 11: Simulation Notes for Dark Matter Characteristics for Cosme.

\begin{tabular}{|c|c|c|}
\hline SI No: & Simulation Specifications & Value \\
\hline 1 & x range (WIMP mass) & 1 (lower bound) \\
\hline 2 & $\begin{array}{c}\text { x range (WIMP mass) } \\
\text { y range (cross section) } \\
\text { smallest }\end{array}$ & c-s: $10^{\wedge}-54 \mathrm{~cm}^{\wedge} 2$ \\
\hline 3 & $\begin{array}{c}\text { c^ } 2 \\
\text { largest }\end{array}$ & c-s: $10^{\wedge}-26 \mathrm{~cm}^{\wedge} 2$ \\
\hline 4 & $\begin{array}{c}\text { y range (cross section) } \\
\text { Type of method }\end{array}$ & Experiment \\
\hline 5 & Limit & Coup Independent \\
\hline 7 & Type & All \\
\hline 8 & Year & All \\
\hline 9 & Simulation Type & \\
\hline
\end{tabular}

Table 12: Simulation Notes for Dark Matter Characteristics For CREST-I.

\begin{tabular}{|c|c|c|}
\hline SI No: & Simulation Specifications & Value \\
\hline 1 & x range (WIMP mass) & 1 (lower bound) \\
\hline 2 & x range (WIMP mass) & $\begin{array}{c}10000 \text { (upper bound) GeV/ } \\
\mathrm{c}^{\wedge} 2\end{array}$ \\
\hline 3 & $\begin{array}{c}\text { y range (cross section) } \\
\text { smallest }\end{array}$ & c-s: $10^{\wedge}-54 \mathrm{~cm}^{\wedge} 2$ \\
\hline 4 & $\begin{array}{c}\text { y range (cross section) } \\
\text { largest }\end{array}$ & c-s: $10^{\wedge}-26 \mathrm{~cm}^{\wedge} 2$ \\
\hline 5 & Type of method & Experiment \\
\hline 6 & Limit & Spin Independent \\
\hline 7 & Type & CREST-I \\
\hline 8 & Year & All \\
\hline 9 & Simulation Type & All \\
\hline
\end{tabular}

Table 13: simulation notes for dark matter characteristics for cuoricino.

\begin{tabular}{|c|c|c|}
\hline SI No: & Simulation Specifications & Value \\
\hline 1 & x range (WIMP mass) & 1 (lower bound) \\
\hline 2 & $\begin{array}{c}\text { x range (WIMP mass) } \\
\text { (10000 (upper bound) } \mathrm{GeV} / \\
\mathrm{c}^{\wedge} 2\end{array}$ \\
\hline 3 & $\begin{array}{c}\text { y range (cross section) } \\
\text { smallest }\end{array}$ & c-s: $10^{\wedge}-54 \mathrm{~cm}^{\wedge} 2$ \\
\hline 4 & $\begin{array}{c}\text { y range (cross section) } \\
\text { largest }\end{array}$ & c-s: $10^{\wedge}-26 \mathrm{~cm}^{\wedge} 2$ \\
\hline 5 & Method & Projection \\
\hline 6 & Limit & Spin Independent \\
\hline 7 & Type & CUORICINO \\
\hline 8 & Year & All \\
\hline 9 & Simulation Type & All \\
\hline
\end{tabular}

Table 14: Simulation Notes for Dark Matter Characteristics for CoGeNT.

\begin{tabular}{|c|c|c|}
\hline Sl No: & Simulation Specifications & Value \\
\hline 1 & $\mathrm{x}$ range (WIMP mass) & 1 (lower bound) \\
\hline 2 & $\mathrm{x}$ range (WIMP mass) & 10000 (upper bound) $\mathrm{GeV} /$ \\
\hline 3 & $\begin{array}{c}\text { y range (cross section) } \\
\text { smallest }\end{array}$ & c-s: $10^{\wedge}-54 \mathrm{~cm}^{\wedge} 2$ \\
\hline 4 & $\begin{array}{c}\text { y range (cross section) } \\
\text { largest }\end{array}$ & c-s: $10^{\wedge}-26 \mathrm{~cm}^{\wedge} 2$ \\
\hline 5 & Method & Theory \\
\hline 6 & Limit & Spin Independent \\
\hline 7 & Type & CoGeNT \\
\hline 8 & Year & All \\
\hline 9 & Simulation Type & All \\
\hline
\end{tabular}


Table 15: Simulation Notes for Dark Matter Characteristics for Cuore.

\begin{tabular}{|c|c|c|}
\hline SI No: & Simulation Specifications & Value \\
\hline 1 & x range (WIMP mass) & 1 (lower bound) \\
\hline 2 & x range (WIMP mass) & $\begin{array}{c}10000 \text { (upper bound) GeV/ } \\
\mathrm{c}^{\wedge} 2\end{array}$ \\
\hline 3 & $\begin{array}{c}\text { y range (cross section) } \\
\text { smallest }\end{array}$ & c-s: $10^{\wedge}-54 \mathrm{~cm}^{\wedge} 2$ \\
\hline 4 & $\begin{array}{c}\text { y range (cross section) } \\
\text { largest }\end{array}$ & c-s: $10^{\wedge}-26 \mathrm{~cm}^{\wedge} 2$ \\
\hline 5 & Method & Experiment \\
\hline 6 & Limit & Spin Independent \\
\hline 7 & Type & Cuore \\
\hline 8 & Year & All \\
\hline 9 & Simulation Type & All \\
\hline
\end{tabular}

Table 16: Simulation notes for dark matter characteristics for dama.

\begin{tabular}{|c|c|c|}
\hline Sl No: & Simulation Specifications & Value \\
\hline 1 & x range (WIMP mass) & 1 (lower bound) \\
\hline 2 & $\mathrm{x}$ range (WIMP mass) & $\begin{array}{c}10000 \text { (upper bound) GeV/ } \\
c^{\wedge} 2\end{array}$ \\
\hline 3 & $\begin{array}{l}\text { y range (cross section) } \\
\text { smallest }\end{array}$ & c-s: $10^{\wedge}-54 \mathrm{~cm}^{\wedge} 2$ \\
\hline 4 & $\begin{array}{c}\text { y range (cross section) } \\
\text { largest }\end{array}$ & c-s: $10^{\wedge}-26 \mathrm{~cm}^{\wedge} 2$ \\
\hline 5 & Method & Experiment \\
\hline 6 & Limit & Spin dependent \\
\hline 7 & Type & DAMA \\
\hline 8 & Year & All \\
\hline 9 & Simulation Type & All \\
\hline
\end{tabular}

Table 17: Simulation Notes for Dark Matter Characteristics for DAMIC.

\begin{tabular}{|c|c|c|}
\hline SI No: & Simulation Specifications & Value \\
\hline 1 & x range (WIMP mass) & 1 (lower bound) \\
\hline 2 & $\begin{array}{c}\text { x range (WIMP mass) } \\
\text { (W } \begin{array}{c}10000 \text { (upper bound) GeV/ } \\
\mathrm{c}^{\wedge} 2\end{array}\end{array}$ \\
\hline 3 & $\begin{array}{c}\text { y range (cross section) } \\
\text { smallest }\end{array}$ & c-s: $10^{\wedge}-54 \mathrm{~cm}^{\wedge} 2$ \\
\hline 4 & $\begin{array}{c}\text { y range (cross section) } \\
\text { largest }\end{array}$ & c-s: $10^{\wedge}-26 \mathrm{~cm}^{\wedge} 2$ \\
\hline 5 & Method & ALL \\
\hline 6 & Limit & DAMIC Independent \\
\hline 7 & Type & All \\
\hline 8 & Year & All \\
\hline 9 & Simulation Type & \\
\hline
\end{tabular}

Table 18: Simulation Notes for Dark Matter Characteristics for DMTPC.

\begin{tabular}{|c|c|c|}
\hline SI No: & Simulation Specifications & Value \\
\hline 1 & x range (WIMP mass) & 1 (lower bound) \\
\hline 2 & x range (WIMP mass) & $\begin{array}{c}10000 \text { (upper bound) GeV/ } \\
\text { c }^{\wedge}\end{array}$ \\
\hline 3 & $\begin{array}{c}\text { y range (cross section) } \\
\text { smallest }\end{array}$ & c-s: $10^{\wedge}-54 \mathrm{~cm}^{\wedge} 2$ \\
\hline 4 & $\begin{array}{c}\text { y range (cross section) } \\
\text { largest }\end{array}$ & c-s: $10^{\wedge}-26 \mathrm{~cm}^{\wedge} 2$ \\
\hline 5 & Method & Projection \\
\hline 6 & Limit & ALL \\
\hline 7 & Type & DAMIC \\
\hline 8 & Year & All \\
\hline 9 & Simulation Type & All \\
\hline
\end{tabular}

Table 19: Simulation notes for dark matter characteristics for drift.

\begin{tabular}{|c|c|c|}
\hline SI No: & Simulation Specifications & Value \\
\hline 1 & x range (WIMP mass) & 1 (lower bound) \\
\hline 2 & x range (WIMP mass) & $\begin{array}{c}10000 \text { (upper bound) GeV/ } \\
\text { c }^{\wedge} 2\end{array}$ \\
\hline 3 & $\begin{array}{c}\text { y range (cross section) } \\
\text { smallest }\end{array}$ & c-s: $10^{\wedge}-54 \mathrm{~cm}^{\wedge} 2$ \\
\hline 4 & $\begin{array}{c}\text { y range (cross section) } \\
\text { largest }\end{array}$ & c-s: $10^{\wedge}-26 \mathrm{~cm}^{\wedge} 2$ \\
\hline 5 & Method & Projection \\
\hline 6 & Limit & ALL \\
\hline 7 & Type & DRIFT \\
\hline 8 & Year & All \\
\hline 9 & Simulation Type & All \\
\hline
\end{tabular}

Table 20: Simulation Notes for Dark Matter Characteristics for Dark Side.

\begin{tabular}{|c|c|c|}
\hline Sl No: & Simulation Specifications & Value \\
\hline 1 & $\mathrm{x}$ range (WIMP mass) & 1 (lower bound) \\
\hline 2 & $\mathrm{x}$ range (WIMP mass) & 10000 (upper bound) $\mathrm{GeV} /$ \\
\hline 3 & $\begin{array}{c}\text { y range (cross section) } \\
\text { smallest }\end{array}$ & $\mathrm{c}-\mathrm{s}: 10^{\wedge}-54 \mathrm{~cm}^{\wedge} 2$ \\
\hline 4 & $\begin{array}{c}\text { y range (cross section) } \\
\text { largest }\end{array}$ & $\mathrm{c}-\mathrm{s}: 10^{\wedge}-26 \mathrm{~cm}^{\wedge} 2$ \\
\hline 5 & Method & Experiment \\
\hline 6 & Limit & Spin independent \\
\hline 7 & Type & Dark Side \\
\hline 8 & Year & All \\
\hline 9 & Simulation Type & All \\
\hline
\end{tabular}


c) Dark Matter Simulation for CDEX-10 (Figures 10)

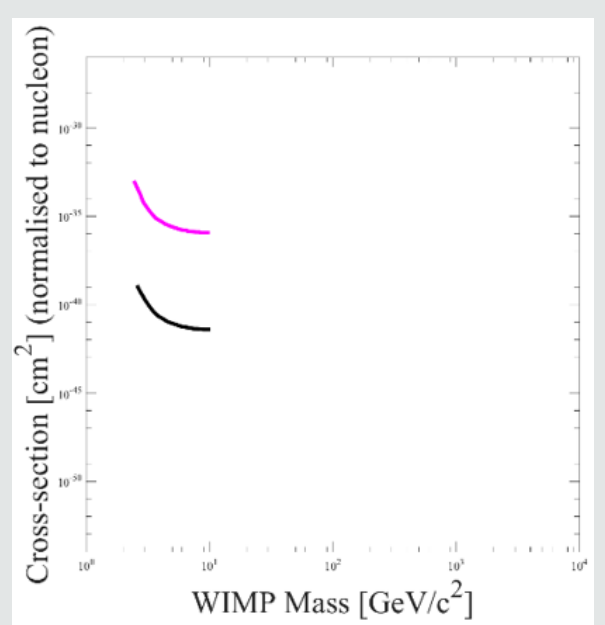

Figure 10: Illustrates characteristics of Dark Matter for CDEX-10.

d) Dark Matter Simulation for CDEX-10(Figures 11)

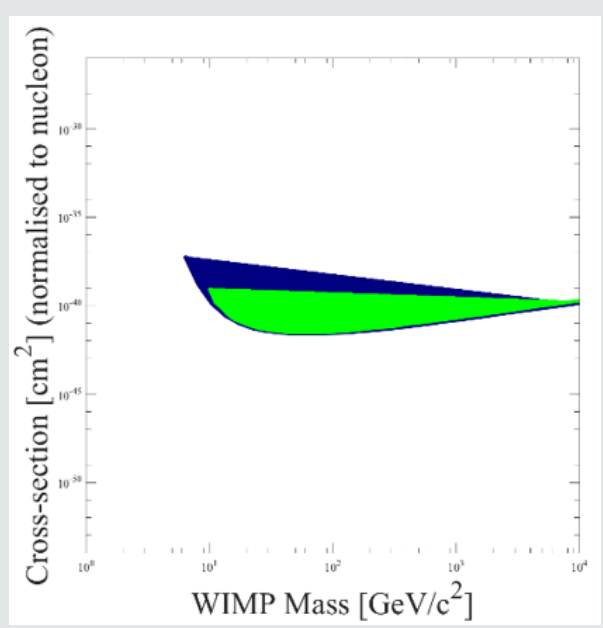

Figure 11: Illustrates characteristics of Dark Matter for CDMS I (SUF).

e) Dark Matter Simulation for CDMS II (Soudan) (Figures 12)

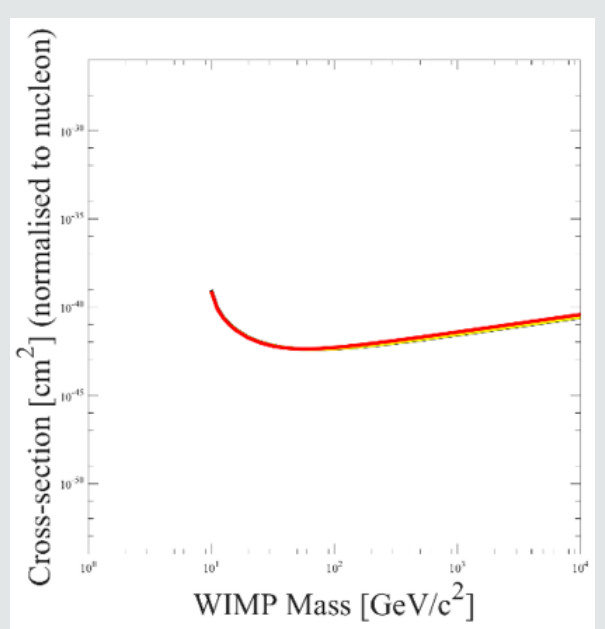

Figure 12: Illustrates characteristics of Dark Matter for CDMS II (Soudan). f) Dark Matter Simulation for COSME (Figures 13)

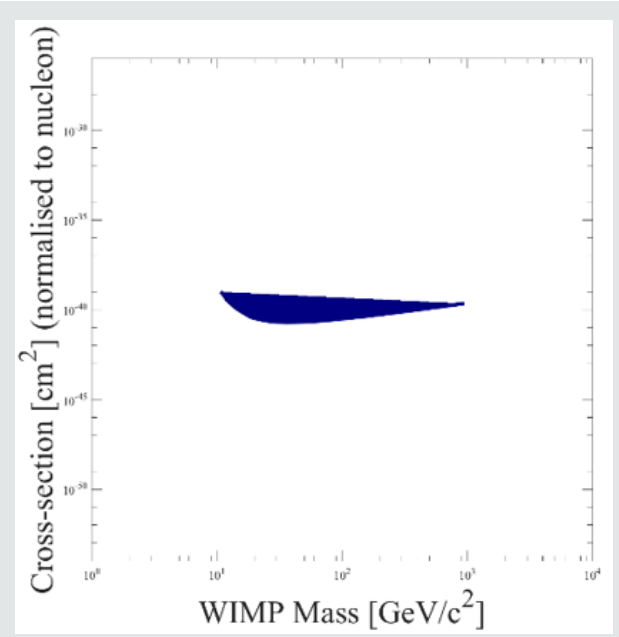

Figure 13: Illustrates characteristics of Dark Matter for COSME.

g) Dark Matter Simulation for COUPP (Figures 14)

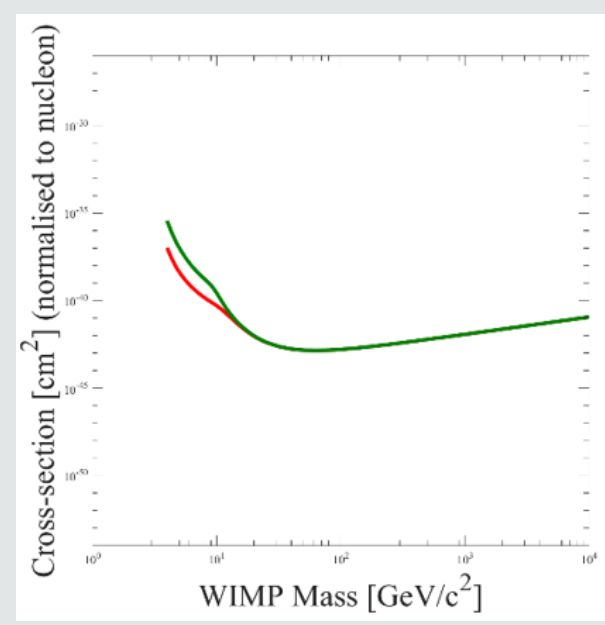

Figure 14: Illustrates characteristics of Dark Matter for COUPP.

h) Dark Matter Simulation for CREST-I (Figures 15)

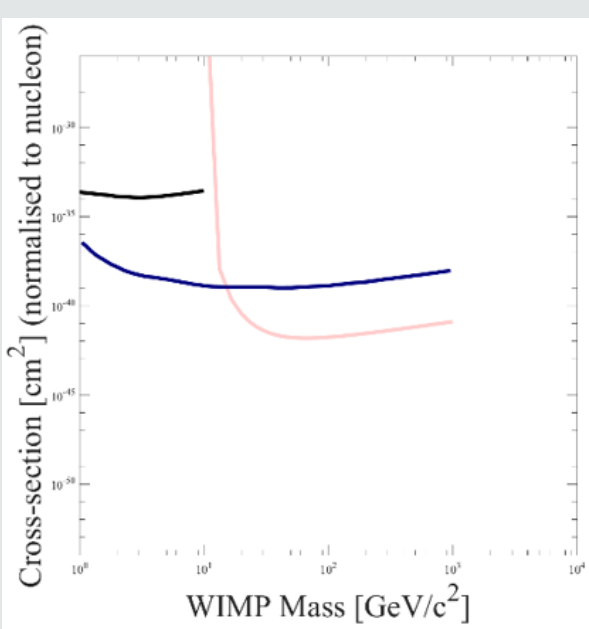

Figure 15: Illustrates characteristics of Dark Matter for CREST-I. 
i) Dark Matter Simulation for CUORICINO (Figures 16)

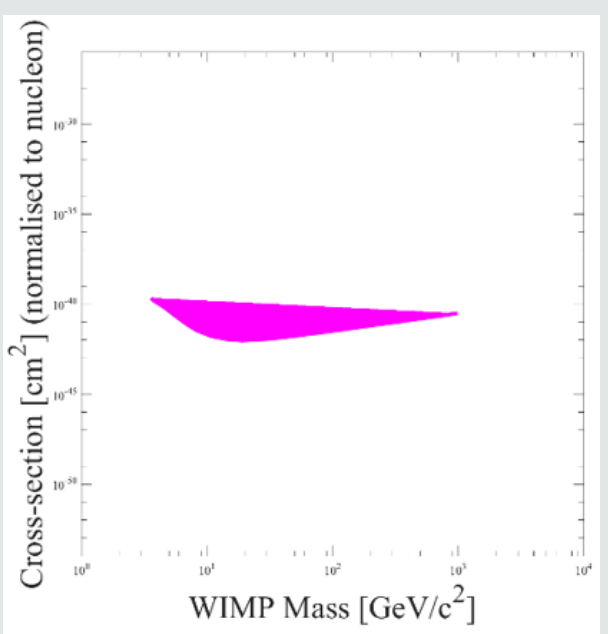

Figure 16: Illustrates characteristics of Dark Matter for CUORICINO.

j) Dark Matter Simulation for CoGeNT (Figures 17)

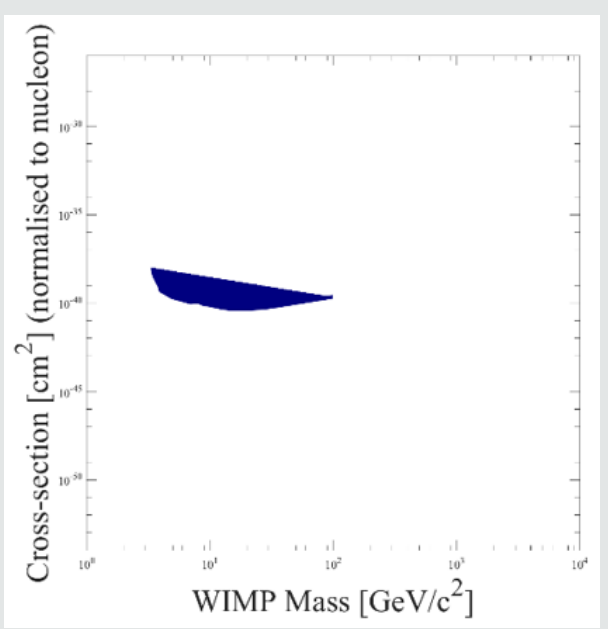

Figure 17: Illustrates characteristics of Dark Matter for CoGeNT.

k) Dark Matter Simulation for Cuore (Figures 18)

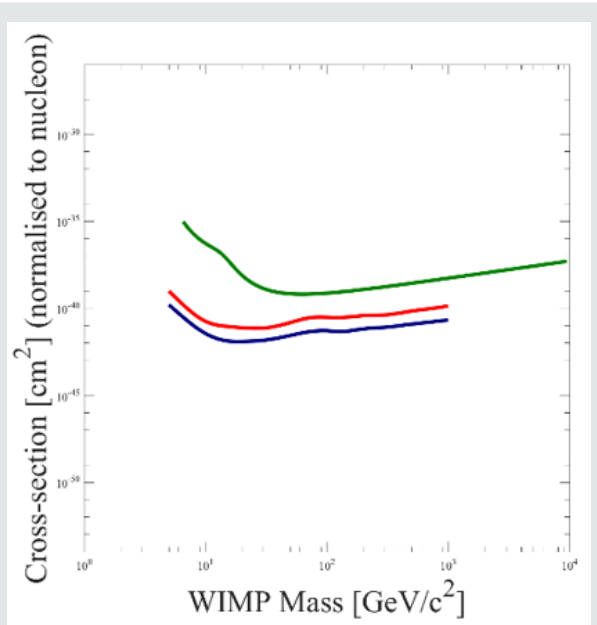

Figure 18: Illustrates characteristics of Dark Matter for Cuore. l) Dark Matter Simulation for DAMA (Figures 19)

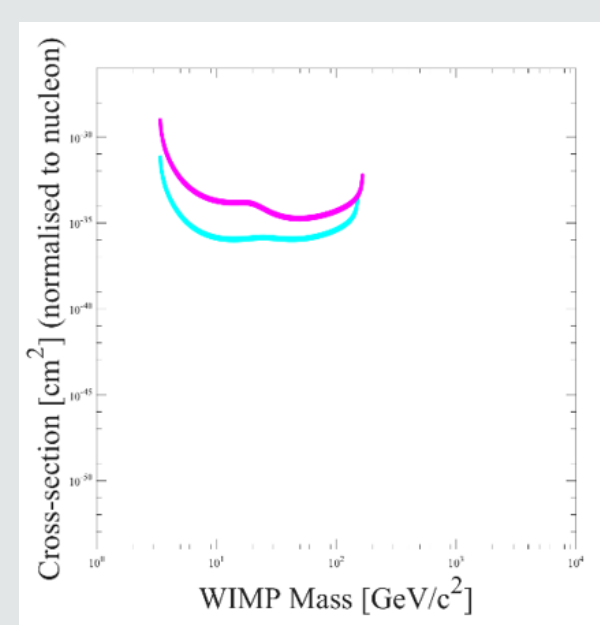

Figure 19: Illustrates characteristics of Dark Matter for DAMA.

m) Dark Matter Simulation for DAMIC (Figures 20)

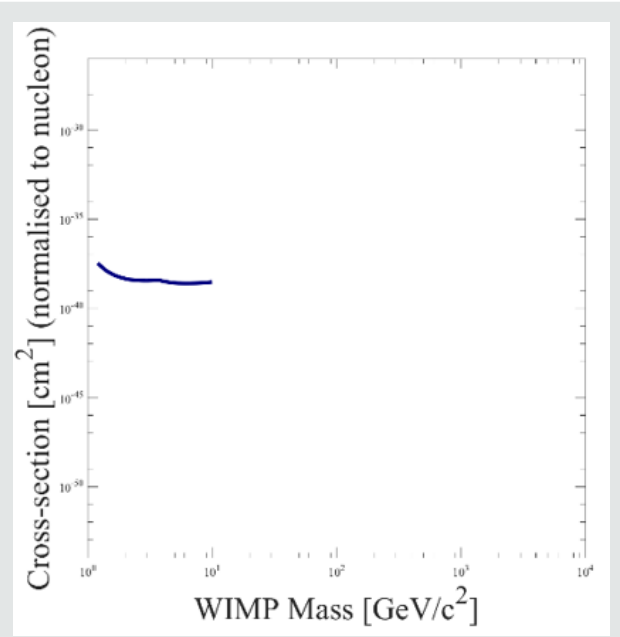

Figure 20: Illustrates characteristics of Dark Matter for DAMIC.

n) Dark Matter Simulation for DMTPC (Figures 21)

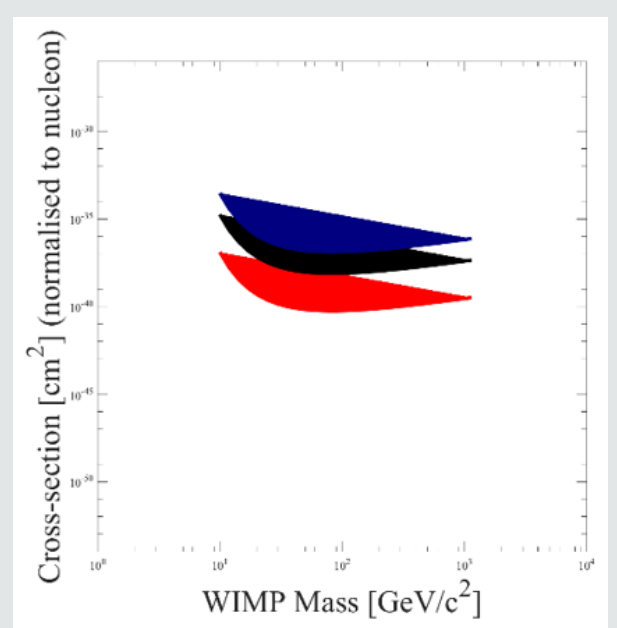

Figure 21: Illustrates characteristics of Dark Matter for DMTPC. 
o) Dark Matter Simulation for DRIFT (Figures 22)

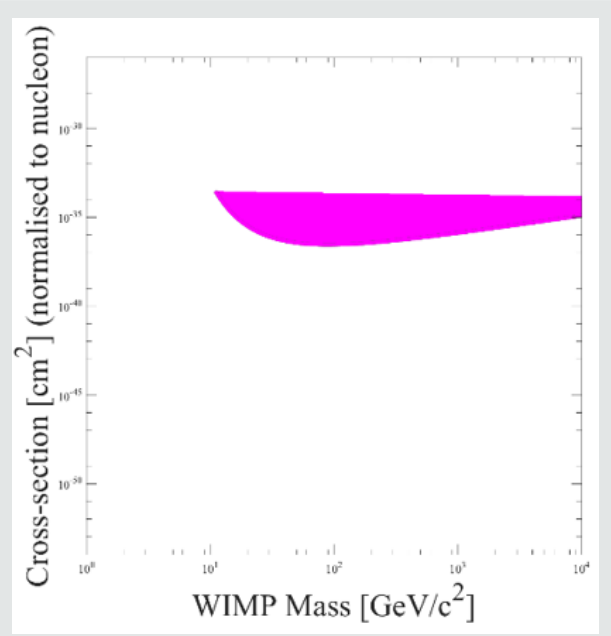

Figure 22: Illustrates characteristics of Dark Matter for DRIFT.

p) Dark Matter Simulation for Dark Side (Figures 23)

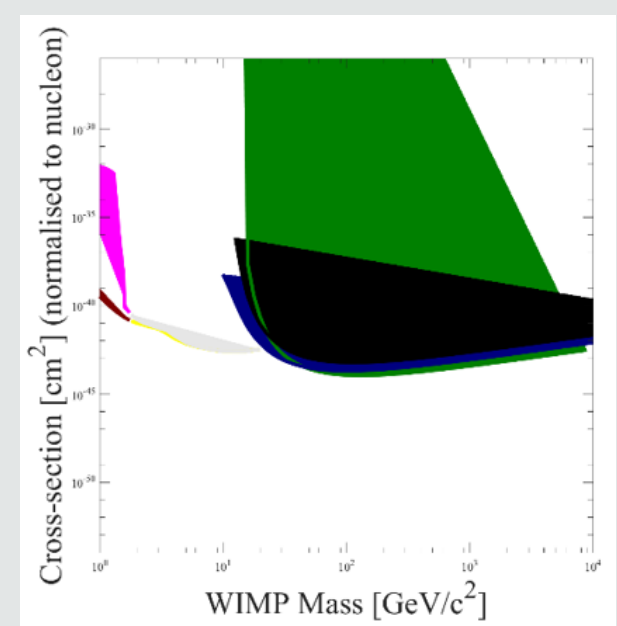

Figure 23: Illustrates characteristics of Dark Matter for Dark Side.

\section{Results}

What is claimed in this research article are

a. Various Plots for Dark Matter have been plotted for spin dependent, spin independent

b. Plots for CDEX-10, CDMS I (SUF), CDMS II (Soudan), COSME has been depicted

c. COUPP graph has been plotted and values has been illustrated in the table d. CREST-I graph has been plotted and values has been illustrated in the table

e. CUORICI graph has been plotted and values has been illustrated in the table

f. CoGeNT graph has been plotted and values has been illustrated in the table

g. Cuore graph has been plotted and values has been illustrated in the table

h. DAMA graph has been plotted and values has been illustrated in the table

i. DAMIC graph has been plotted and values has been illustrated in the table

j. DMPTC graph has been plotted and values has been illustrated in the table

k. DRIFT graph has been plotted and values has been illustrated in the table

1. Dark Side graph has been plotted and values has been illustrated in the table

\section{Conclusion}

To conclude various samples from CDEX-10, CDMS I (SUF), CDMS II (Soudan), COSME, COUPP, CREST-I, CUORICI, CoGeNT, Cuore, DAMA, DAMIC, DMPTC, DRIFT, Dark Side has been illustrated with theoretical, experiment with various limits of spin dependent and spin independent.

\section{Discussion}

In this research article all the values are taken as default values which are documented in Tables. Results would vary with the change of $x$ range (WIMP mass), $y$ range (cross section), etc.

\section{Acknowledgment}

Author would like to thank Prof. Navarun Gupta, Prof. Hassan Bajwa, Prof. Linfeng Zhang and Prof. Hmurcik for their academic support. Author also thanks anonymous reviewers for their comments.

\section{Conflicts of Interest}

There is no conflict of interest as per Author's point of view.

\section{References}

1. (2011) Dark matter science. Physics Org.

2. Redd NT (2017) What is Dark Matter?

3. Desai A, Moskowitz A (2013) Tools Limit Plot generator. 
CC) (P) This work is licensed under Creative

To Submit Your Article Click Here: Submit Article

DOI: 10.32474/MAMS.2019.01.000115

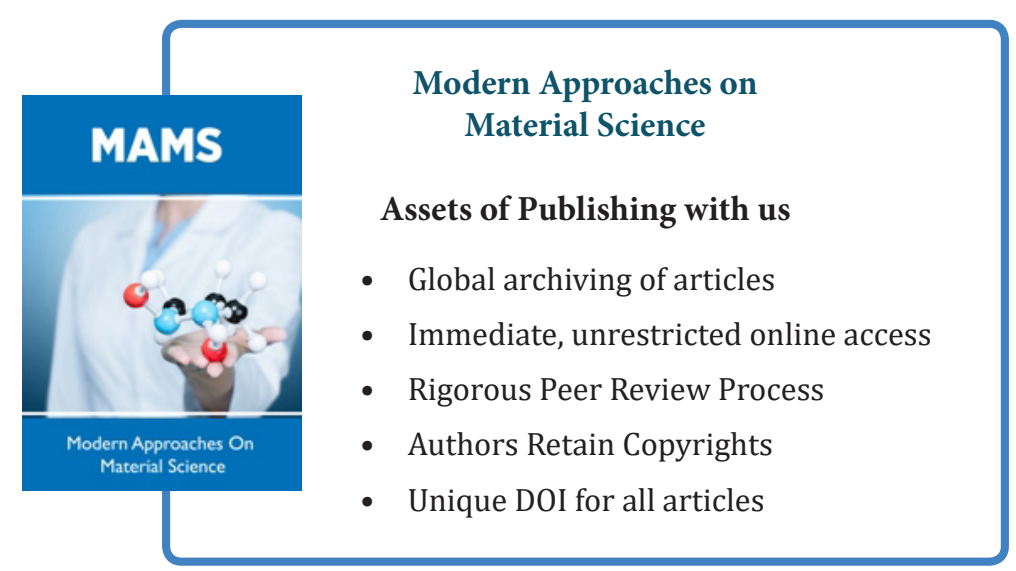

